\title{
Results on the Analyticity of Many-Body Scattering Amplitudes in Perturbation Theory
}

\author{
Paul Federbush \\ Department of Mathematics, University of Michigan, Ann Arbor, Michigan
}

(Received 8 February 1967)

\begin{abstract}
The problem of analytic continuation of the many-body scattering amplitude associated with a perturbation-theory diagram under the rotation of the final momenta from real to complex momenta, $\mathbf{k} \rightarrow(1+i \theta) \mathbf{k}$, is studied. It is shown that the contour of integration over internal momenta can be distorted avoiding singularities of the integrand, as $\theta$ varies for small enough $\theta$. If the diagram is connected enough, the potentials are Yukawa-type, $\operatorname{Re} E>0$, and $\operatorname{Im} E<0$. The rotation angle can be picked independently of $\operatorname{Im} E$.
\end{abstract}

\section{INTRODUCTION}

$\mathrm{W}^{\mathrm{E}}$ E intend to study the analytic continuation in $\theta$ of a many-body scattering diagram, where the final momenta, all initially real, are rotated under the transformation $\mathbf{k} \rightarrow(1+i \theta) \mathbf{k}$. A more general type of continuation will also be studied. We restrict our attention to $\operatorname{Re} E>0, \operatorname{Im} E<0$, the most interesting situation. The heart of the study is an analysis of a peculiar geometrical problem. The type of geometrical analysis involved clearly can be applied to more general problems than those studied here, about which a few comments will be made later.

\section{SCATTERING DIAGRAM}

We deal with an $N$-particle scattering situation. There will then be $N$ masses, $M_{1}, M_{2}, \cdots, M_{N}$, and a Hamiltonian

$$
H=\sum_{i} \frac{\mathbf{k}_{i}^{2}}{2 M_{i}}+\sum_{i<j} V_{i j}\left(\left|\mathbf{x}_{i}-\mathbf{x}_{j}\right|\right) .
$$

Clearly we limit ourselves to two-body interactions. We change momentum variables so that $H_{0}$ can be written

$$
H_{0}=\sum_{i} \mathbf{k}_{i}^{2},
$$

and there is then the equation of momentum conservation

$$
\sum_{i} \mathbf{k}_{i} M_{i}^{\frac{1}{2}}=\text { const. }
$$

Now consider a perturbation-theory diagram specified by a sequence of interactions and internal momenta (before integration). We write the momentum of each state as a vector in $3 N$-dimensional Euclidean space $\left(\mathbf{k}_{1}, \mathbf{k}_{2}, \cdots, \mathbf{k}_{N}\right)$. By working in the center-of-mass system, one can take

$$
\sum_{i} M_{i}^{\frac{1}{2}} \mathbf{k}_{i}=0
$$

If there are $S$ intermediate states in the diagram, there is then the following sequence of $(S+2)$ vectors:

$$
\begin{gathered}
\left(\mathbf{k}_{1}^{\text {in }}, \mathbf{k}_{2}^{\text {in }}, \cdots, \mathbf{k}_{N}^{\text {in }}\right) \\
\left(\mathbf{k}_{1}^{1}, \mathbf{k}_{2}^{1}, \cdots, \mathbf{k}_{N}^{1}\right) \\
\cdot \\
\cdot \\
\left(\mathbf{k}_{1}^{s}, \mathbf{k}_{2}^{s}, \cdots, \mathbf{k}_{N}^{s}\right) \\
\left(\mathbf{k}_{1}^{\text {fin }}, \mathbf{k}_{2}^{\text {fin }}, \cdots, \mathbf{k}_{N}^{\text {fin }}\right) .
\end{gathered}
$$

For each of the vectors, Eq. (4) holds. If the interaction between the $i$ th intermediate state and the $(i+1)$ th intermediate state is $V_{L T}$, the vector difference in $3 N$-dimensional space

$$
\left(\mathbf{k}_{1}^{i+1}, \cdots, \mathbf{k}_{N}^{i+1}\right)-\left(\mathbf{k}_{1}^{i}, \cdots, \mathbf{k}_{N}^{i}\right)
$$

is parallel to the vector

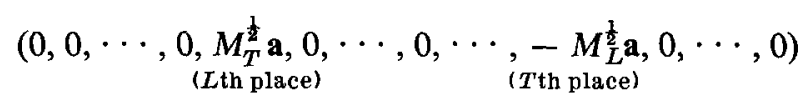

for some three-dimensional vector a. We prefer to think of this difference as the appropriate succession of three displacements in the directions

$$
\begin{aligned}
& \left(0,0, \cdots, M_{T}^{\frac{1}{2}} \mathbf{i}, 0, \cdots, 0,-M_{L}^{\frac{1}{2}} \mathbf{i}, 0, \cdots, 0\right), \\
& \left(0,0, \cdots, M_{T}^{\frac{1}{2}} \mathbf{j}, 0, \cdots, 0,-M_{L}^{\frac{1}{L}} \mathbf{j}, 0, \cdots, 0\right), \\
& \left(0,0, \cdots, M_{T}^{\frac{1}{2}} \mathbf{k}, 0, \cdots, 0,-M_{L}^{\frac{1}{2}} \mathbf{k}, 0, \cdots, 0\right) .
\end{aligned}
$$

Thus we have a $3(N-1)$-dimensional vector space (the subspace of $E^{3 N}$ with $\sum M_{i}^{\frac{1}{2}} \mathbf{k}_{i}=0$ ), and a sequence of $4+3 S$ vectors in this space (breaking the momentum transfer at each interaction into a sequence of three momentum transfers, as indicated above). In the sequence, two successive vectors differ by a vector parallel to one of the $3[N(N-1) / 2]$ possible directions (three directions are associated to every interaction). These $3[N(N-1) / 2]$ directions will be called preferred directions.

The integration over internal momenta is an integration over all possible sequences of $3 S+2$ 
vectors consistent with the direction restrictions associated with the interactions.

We define, in the usual sense, the connectivity properties of the perturbation diagram. The diagram is $M$-connected if the sequence of $3 S+3$ vectors can be partitioned into $M$ subsequences, and at most $M$ subsequences, such that the vectors in each subsequence are a spanning set. (The sequence of $3 S+3$ vectors is the sequence of preferred directions associated with the diagram.) This is equivalent to being able to subdivide the diagram into a sequence of $M$ diagrams, and at most $M$ diagrams, such that each subdiagram is connected.

As we will be concerned with analytic continuation, we will allow the momenta to become complex. We restrict our attention to distortions of the original real contour that project one-to-one onto the original contour under the projection that sends each complex momentum onto its real part. Call these semiflat contours. Such a contour is specified by associating with each sequence of $3 S+2$ real vectors in the $3(N-1)$-dimensional space, subject to the conditions that successive vector differences are parallel to the appropriate preferred directions, a second sequence of $3 S+2$ vectors (the imaginary parts of the momenta), with the same conditions on successive vector differences.

The contour of integration may be distorted in any bounded region provided the integrand is analytic through the region of distortion. We will also allow distortions of the contour at infinity, a procedure that must be studied separately. The analyticity of the integrand involves the analyticity of the potentials and the analyticity of the energy denominators. For the first result we aim at below, we require the analyticity properties of the potential in momentum space to be that of a superposition of Yukawa potentials with a minimum mass greater than zero. We will also give a similar result for the case of momentum-space analyticity of the potentials in a strip about the real momentum axis as is obtained if, in coordinate space,

$$
|V(x)|<c e^{-\alpha|x|}, \quad \alpha>0
$$

for some $c$ and $\alpha$. The essential difficulties and interest arise from consideration of analyticity properties of the energy denominators.

The two results we obtain are among many that are obtainable by a similar procedure. In the conclusion we will indicate further directions. The type of theorems we now aim at are chosen through a consideration of those that seem most useful in the study of many-body scattering theory along the lines of
Faddeev's study of the three-body system. ${ }^{1}$ The type of rotation of the momentum we consider in the theorems below, applied in a more general context, (outside of perturbation theory) could (hopefully) provide a method for dealing with the singular limit of the energy approaching the real axis.

\section{GEOMETRICAL THEOREMS}

We consider the following basic situation, motivated as an abstraction of the considerations of the last section. There is a Euclidean space $E$ and a finite set $P$ of unit vectors in $E$ (specifying preferred directions) that together span $E$.

Lemma 1: There is a constant $c$ such that if any vector $\mathbf{v}$ in $E$ is expressed as a linear combination of linearly independent vectors, $\mathbf{a}_{1}, \mathbf{a}_{2}, \cdots, \mathbf{a}_{g}$ from $P$, each of the expansion coefficients is less than $c|v|$.

\section{Proof: Writing}

$$
\mathbf{v}=\alpha_{1} \mathbf{a}_{1}+\cdots+\alpha_{g} \mathbf{a}_{g},
$$

we take the inner product of $v$ with each of the unit vectors $\mathbf{a}_{1}, \cdots, \mathbf{a}_{0}$ :

$$
\mathbf{a}_{i} \cdot \mathbf{v}=\sum_{k} \alpha_{k} \mathbf{a}_{i} \cdot \mathbf{a}_{k}, \quad i=1,2, \cdots, g .
$$

This set of equations has a unique solution for the $\mathbf{a}_{k}$, since the matrix of inner products $\left(\mathbf{a}_{i} \cdot \mathbf{a}_{k}\right)$ is nonsingular. Because the value of the determinants of all such matrices formed from linearly independent subsets of $P$, being finite in number, is bounded away from zero, the lemma follows.

Lemma 2: Let $\mathbf{u}$ be an arbitrary unit vector in $E$, and let $\mathbf{a}_{1}, \mathbf{a}_{2}, \cdots, \mathbf{a}_{g}$ be any subset of vectors from $P$. Let

$$
\mu=\max _{i=1, \cdots, g}\left(\left|\mathbf{u} \cdot \mathbf{a}_{i}\right|\right),
$$

and let $\theta$ be the angle between $\mathbf{u}$ and the subspace spanned by $a_{1}, \cdots, a_{g}$ (i.e., the smallest angle possible between $u$ and a vector in the subspace). Then

$$
|\cos \theta| \leq g c \mu,
$$

with $c$ the constant of Lemma 1 .

Proof: This follows immediately from Lemma 1 upon writing any vector in the subspace as a combination of the $\mathbf{a}_{k}$.

\footnotetext{
${ }^{1}$ L. D. Faddeev, "Mathematical Problems of the Quantum Theory of Scattering for a Three Particle System," AERE-Trans
} 1002, United Kingdom Atomic Energy Authority Translation. 
Lemma 2': There is a minimum angle between the subspaces spanned by any subset of $P$ and any vector from $P$ not in the subspace.

Proof: This follows since there are only a tinite number of such pairs of subspaces and vectors not in the subspace formable from $P$.

Main Theorem: Let $\left(\mathbf{a}_{\alpha(1)}, \mathbf{a}_{\alpha(2)}, \cdots, \mathbf{a}_{\alpha(g)}\right)$ be a sequence of vectors from the set $P$, possibly with repetitions. The connectivity of this sequence is defined as before as the greatest number of subsequences the sequence can be partitioned into:

$$
\begin{aligned}
\left(\mathbf{a}_{\alpha(1)}, \mathbf{a}_{\alpha(2)}, \cdots, \mathbf{a}_{\alpha[\beta(1)]}\right)\left(\mathbf{a}_{\alpha[\beta(1)+1]}, \cdots\right) \cdots \\
\left(\mathbf{a}_{\alpha[\beta(M-1)+1]}, \cdots, \mathbf{a}_{\alpha(g)}\right),
\end{aligned}
$$

such that each subsequence contains a spanning subset. Let $\left(x_{1}, x_{2}, \cdots, x_{p+1}\right)$ be a sequence of vectors from $E$ with the property that $\mathbf{x}_{i+1}-\mathbf{x}_{i}$ is parallel to $\mathbf{a}_{\alpha(i)}$. Let $a$ be a fixed number greater than zero. There is a $\delta>0$ and an $M_{0}$ such that, for every two sequences related as the above, there is a sequence $\left(\mathbf{y}_{1}, \mathbf{y}_{2}, \cdots, \mathbf{y}_{g+1}\right)$ of vectors with
(a) $\mathbf{y}_{i+1}-y_{i}$ parallel to $a_{\alpha(i)}$,
(b) $\mathbf{y}_{1}=0$,

$$
y_{o+1}=x_{p+1}
$$$$
\text { (c) } \mathbf{y}_{i} \cdot \mathbf{x}_{i} \geq 0 \text { if } a-\delta \leq\left|\mathbf{x}_{i}\right| \leq a+\delta \text {, }
$$

provided the connectivity of the a sequence is greater than $M_{0}$.

It is easy to show that, in general, the theorem would not be true if instead of (c) above we tried

$$
\text { (c') } \mathbf{y}_{i} \cdot \mathbf{x}_{i} \geq 0 \text { if }\left|\mathbf{x}_{i}\right| \leq a \text {. }
$$

However, it does not seem difficult to modify the proof to include the stronger theorem obtained with (c) replaced by

$$
\text { (c) } \mathbf{y}_{i} \cdot \mathbf{x}_{i} \geq 0 \text { if } 0<b \leq\left|\mathbf{x}_{i}\right| \leq a \text {, }
$$

$a$ and $b$ given, limited only by $0<b \leq a$. This generalization, important for some further applications, is not explored here.

Lemma 3: If $\left|\mathbf{x}_{i}-\mathbf{y}_{i}\right|<a-\delta$, the inner product condition (c) is automatically satisfied.

Proof: This is immediate.

Lemma 4: If $\mathrm{x}_{1}, \mathrm{x}_{2}, \cdots, \mathrm{x}_{\beta(1)}$ all lie outside the shell $a-\delta \leq|\mathbf{x}| \leq a+\delta \quad\left[\left(\mathbf{a}_{\alpha(1)}, \cdots, \mathbf{a}_{\alpha[\beta(1)}\right) \quad\right.$ a spanning set as indicated in the statement of the theorem], then $y_{i}$ can be chosen equal to $x_{i}$ for $i=$ $\beta_{1}+1, \beta_{1}+2, \cdots, g$ and $y_{1}, \cdots, y_{\beta(1)}$ can be chosen so that this set of $y_{i}$ satisfies conditions of the theorem.
Proof: Expand $\mathbf{x}_{\beta(1)+1}$ in terms of $\mathbf{a}_{1}, \cdots, \mathbf{a}_{\alpha[\beta(1)]}$,

Pick

$$
\mathbf{x}_{\beta(1)+1}=\alpha_{1} \mathbf{a}_{1}+\cdots+\alpha_{\beta(1)} \mathbf{a}_{\alpha[\beta(1)]} .
$$

$$
\begin{gathered}
y_{1}=0, \quad y_{2}=\alpha_{1} a_{1}, \quad y_{3}=\alpha_{1} a_{1}+\alpha_{2} a_{2}, \\
y_{\beta(1)+1}=x_{\beta(1)+1} .
\end{gathered}
$$

These choices satisfy the necessary conditions.

Lemma 5: There is an $\varepsilon$ such that, if $\left|\mathbf{x}_{i}-\mathbf{y}_{i}\right|<\varepsilon$ for some $i$ and if $a_{\alpha(i)}, \cdots, a_{\alpha(z)}$ form a spanning set, then $y_{s+1}$ can be chosen equal to $x_{s+1}$ and intermediate $y$ 's can be found consistent with the conditions of the theorem.

Proof: Pick a linearly independent set of spanning vectors among $\mathbf{a}_{\alpha(i)}, \cdots, \mathbf{a}_{\alpha(s)}$. Now expand

$$
\mathbf{x}_{i}-\mathbf{y}_{i}=\alpha_{i} \mathbf{a}_{\alpha(i)}+\cdots+\alpha_{s} \mathbf{a}_{\alpha(s)}
$$

with coefficients different from zero only among the linearly independent spanning vectors. Pick

$$
\begin{aligned}
& \mathbf{y}_{i+1}=\mathbf{y}_{i}+\alpha_{i} \mathbf{a}_{\alpha(i)}+\left(\mathbf{x}_{i+1}-\mathbf{x}_{i}\right), \\
& \mathbf{y}_{i+2}=\mathbf{y}_{i+1}+\alpha_{i+1} \mathbf{a}_{\alpha(i+1)}+\left(\mathbf{x}_{i+2}-\mathbf{x}_{i+1}\right), \\
& \mathbf{y}_{s+1}=\mathbf{x}_{s+1} .
\end{aligned}
$$

It follows that

$$
\begin{aligned}
& \mathbf{y}_{i+1}-\mathbf{x}_{i+1}=\left(\mathbf{y}_{i}-\mathbf{x}_{i}\right)+\alpha_{i} \mathbf{a}_{\alpha(i)}, \\
& \mathbf{y}_{i+2}-\mathbf{x}_{i+2}=\left(\mathbf{y}_{i}-\mathbf{x}_{i}\right)+\alpha_{i} \mathbf{a}_{\alpha(i)}+\alpha_{i+1} \mathbf{a}_{\alpha(i+1)} .
\end{aligned}
$$

Thus

$$
\left|\mathbf{y}_{i++}-\mathbf{x}_{i+q}\right| \leq\left|\mathbf{y}_{i}-\mathbf{x}_{i}\right|+\sum\left|\alpha_{i}\right|
$$

Since $\left|\alpha_{i}\right|<c\left|\mathbf{x}_{i}-\mathbf{y}_{i}\right|$ by Lemma 1 , if $D$ is the dimension of the space,

$$
\begin{array}{r}
\left|\mathbf{y}_{i+r}-\mathbf{x}_{i+r}\right| \leq(1+D c)\left|\mathbf{y}_{i}-\mathbf{x}_{i}\right|, \\
r=0,1, \cdots .
\end{array}
$$

If $\left|y_{i}-x_{i}\right|$ is small enough, then $\left|y_{i+r}-x_{i+*}\right|<$ $a-\delta$, which, by Lemma 3 , guarantees that these y's work.

Lemma 6: If $\left|\mathbf{x}_{i}-\mathbf{y}_{i}\right|<a-\delta$ for some $i$, then a sequence of $y$ 's can be found starting with $y_{i}$ which satisfy the conditions of the theorem such that $\left|x_{k}-y_{k}\right|$ is monotonically decreasing; and each time the a's pass through a spanning sequence, the $\left|x_{k}-y_{k}\right|$ decreases at least by some factor $r, r<1 . r$ depends only on the vectors in $P$.

Proof: Pick $\mathbf{y}$ 's successively by minimizing $\left|\mathbf{x}_{k}-\mathbf{y}_{k}\right|$ at each stage; that is, $y_{k+1}-y_{k}=\lambda_{k} a_{\alpha(k)}$ with $\lambda_{k}$ chosen to minimize $\left|x_{k+1}-y_{k+1}\right|$. It is clear that $\left|x_{k}-y_{k}\right|<$ $a-\delta$ for all $k$, so the conditions of the theorem are satisfied. It remains to see that $\left|\mathbf{x}_{k}-\mathbf{y}_{k}\right|$ decreases by some factor as the a's go through a spanning set. We 
omit the proof of this fact; it may be constructed along the lines of the following lemma.

Lemma 7: If $a-\delta \leq\left|\mathbf{x}_{1}\right| \leq a+\delta$ (with $\mathbf{y}_{1}=0$ ), an allowable sequence of $y$ 's can be found such that $\left|\mathbf{x}_{s+1}-\mathbf{y}_{s+1}\right|<a-\delta$, provided $\mathbf{a}_{1}, \cdots, \mathbf{a}_{s}$ are a spanning set (from $P$ ) and $\delta$ is small enough. $\delta$ may be chosen, depending on $a$ and the set $P$, but independently of the $\mathbf{x}$ 's.

The main geometric theorem follows from Lemmas 3-7. If $\mathbf{x}$ never hits the spherical shell as the a's pass through the first spanning set, Lemma 4 shows the existence of the $\mathbf{y}$ 's. If some $\mathbf{x}$ hits the spherical shell as the a's pass through the first spanning set, use Lemma 7 to obtain $\left|\mathbf{x}_{j}-\mathbf{y}_{j}\right|<a=\delta$. Then use Lemma 6 to get $\left|x_{k}-y_{k}\right|$ small enough to use Lemma 5 , completing the sequence of $\mathbf{y}$ 's.

Proof of Lemma 7: Let $D$ be the dimension of the Euclidean space, $\theta_{\text {min }}$ be the angle of Lemma $2^{\prime}$, and $c$ be the constant of Lemma 1 . Choose $\theta_{1}, \theta_{2}, \cdots$, $\theta_{D-1}$ such that

$$
\begin{aligned}
\theta_{1} & =\phi_{1}>0, \\
\theta_{i+1}-2 \sin ^{-1}\left(D c \sin \theta_{i}\right) & =\phi_{i+1}>0, \\
\theta_{\min }-2 \sin ^{-1}\left(D c \sin \theta_{D-1}\right) & =\phi_{D}>0 .
\end{aligned}
$$

Let $\phi_{\min }=\min \left(\phi_{1}, \phi_{2}, \cdots, \phi_{D}\right)$; pick $\delta$ such that $(a+\delta) \cos \phi_{\min }<a-\delta$. By assumption, $a-\delta \leq$ $\left|\mathbf{x}_{1}\right| \leq a+\delta$. Let $\mathbf{a}_{1}, \mathbf{a}_{2}, \cdots, \mathbf{a}_{R}$ be a spanning set, but. $\mathbf{a}_{1}, \mathbf{a}_{2}, \cdots, \mathbf{a}_{R-1}$ not. Let $\mathbf{b}_{1}=\mathbf{a}_{1}, \mathbf{b}_{i}=\mathbf{a}_{\beta(i)}, \mathbf{b}_{i}$ the first of the $\mathbf{a}_{j}$ 's linearly independent of $\mathbf{b}_{1}, \mathbf{b}_{2}, \cdots$, $\mathbf{b}_{i-1}, \mathbf{b}_{D}$ will equal $\mathbf{a}_{R}$. Let $\psi_{i}=\pi / 2-\angle\left(\mathbf{b}_{i}, \mathbf{x}_{1}\right)$. By $\angle\left(b_{i}, x_{1}\right)$ we mean the angle between the vectors $b_{i}$ and $x_{1}$ (minus their orientation), an angle between 0 and $\pi / 2$. Let $\psi_{k}$ be the first of the $\psi$ 's with $\psi_{k} \geq \phi_{k}$. Then $\psi_{1}<\phi_{1}, \psi_{2}<\phi_{2}, \cdots, \psi_{k-1}<\phi_{k-1}$, and $\psi_{k} \geq$ $\phi_{k}$. Such a $k$ must exist due to the nature of the $\phi$ 's. If $\left|\mathbf{x}_{\beta(k)}\right| \leq a+\delta$, let

$$
\mathbf{y}_{\mathbf{1}}=\mathbf{y}_{\mathbf{2}}=\cdots=\mathbf{y}_{\beta(k)}=0, \quad \mathbf{y}_{\beta(k)+1}=\lambda \mathbf{b}_{k k},
$$

$\lambda$ chosen to minimize $\left|\mathbf{x}_{\beta(k)+1}-\mathbf{y}_{\beta(k)+1}\right|$. If $\left|\mathbf{x}_{\beta(k)}\right|>$ $a+\delta$, with

$$
\begin{gathered}
\left|\mathbf{x}_{g}\right| \leq a+\delta, \quad\left|\mathbf{x}_{g+1}\right|>a+\delta, \\
\left|\mathbf{x}_{g+2}\right|>a+\delta, \quad \cdots, \quad\left|\mathbf{x}_{\beta(k)}\right|>a+\delta,
\end{gathered}
$$

then pick

$$
\begin{aligned}
& \mathbf{y}_{1}= \mathbf{y}_{2}=\cdots=\mathbf{y}_{g}=0, \\
& \mathbf{y}_{g+1}= \mathbf{x}_{g+1}-\mathbf{x}_{g}, \\
& \cdot \cdot \\
& \mathbf{y}_{\beta(k)}= \mathbf{x}_{\beta(k)}-\mathbf{x}_{g}, \\
& \mathbf{y}_{\beta(k)+1}=\lambda \mathbf{b}_{k}+\mathbf{y}_{\beta(k)},
\end{aligned}
$$

$\lambda$ chosen to minimize $\left|\mathbf{x}_{\beta(k)+1}-\mathbf{y}_{\beta(k)+1}\right|$. One can check that $\left|\mathbf{y}_{\beta(k)+1}-\mathbf{x}_{\beta(k)+1}\right|<a-\delta$ in both cases.

\section{ANALYTIC CONTINUATION}

Theorem 1: If $E=a^{2}-i \epsilon, a>0, \epsilon>0$, and if all the potentials involved have the analyticity of a superposition of Yukawa potentials with a minimum mass greater than zero, then there is an $M_{0}$ and an $\eta$ such that any perturbation-theory diagram with connectivity $\geq M_{0}$ considered a function of $\theta, \mathbf{k}_{\mathrm{fn}} \rightarrow$ $(1+i \theta) \mathbf{k}_{\mathrm{fn}}\left[\mathbf{k}_{\mathrm{in}}\right.$ and $\mathbf{k}_{\mathrm{fn}}$ being real] has the property that the intermediate-state integration contour can be distorted through analytic regions of the integrand as $\theta$ varies from $\theta=0$ to $\theta=\eta$. $\eta$ will depend on:

(a) $N$, (b) $M_{1}, M_{2}, \cdots, M_{N}$, (c) the minimal mass in the Yukawa decomposition of the potentials, (d) $a$, (e) $\mathbf{k}_{\text {in }}$, but not on $\mathbf{k}_{\text {fin }}$.

It is important to notice that the requirement of high connectivity is essential. For example, with $N=3$, the simple second-order diagram with interactions $V_{12}$ followed by $V_{23}$ cannot be continued as above. With $\mathbf{k}_{\mathrm{in}}$ and $\mathbf{k}_{\mathrm{fn}}$ specified in this case, the possible rotation angle may approach zero as $\epsilon \rightarrow 0$. The existence of the rotation in the limit $\epsilon \rightarrow 0$ is most crucial.

It is reasonable to suppose that by increasing the connectivity requirements the amount of rotation can be increased beyond that allowed by the theorem. This is an interesting question, but possibly not important to the proposed application of the theorem.

To prove Theorem 1, we observe that the following bounds can be put on $\left|\mathbf{y}_{i}\right|$ and $\left|\mathbf{y}_{i+1}-\mathbf{y}_{i}\right|$ by examining the construction of Lemmas 3-7:

$$
\begin{aligned}
\left|\mathbf{y}_{i}\right| & \leq c_{1}\left|\mathbf{x}_{1}\right|+c_{2}\left|\mathbf{x}_{i}\right| \\
\left|\mathbf{y}_{i+1}-\mathbf{y}_{i}\right| & \leq c_{3}\left|\mathbf{x}_{i+1}-\mathbf{x}_{i}\right|+c_{4}\left|\mathbf{x}_{1}\right|
\end{aligned}
$$

We identify the $a$ of the theorem and the $a$ of the geometric construction, and associate the $x$ 's with the real parts of the momenta, the y's with a constant $\lambda$ times the imaginary part of the momenta. It is easy to see that, with a fixed $a$, fixed initial momenta, and minimum mass of the Yukawa potentials, if $\lambda$ is large enough $\left(\lambda \geq \lambda_{0}\right.$, say), the real and imaginary parts form a complex momentum always avoiding the singularities of the potentials and the energy denominators. The inner product condition of the geometric construction guarantees that the denominators are never singular. If $\operatorname{Im} E$ is fixed at some negative value, then the sequences of y's satisfying the conditions of the geometric theorem form a convex set under vector-byvector addition. We can impose (15) and still maintain a convex set of solutions. Since $\operatorname{Im} E<0$, we can 
replace the inner product inequality $\mathbf{x}_{i} \cdot \mathbf{y}_{i} \geq 0$ by $\mathbf{x}_{i} \cdot \mathbf{y}_{i}>-\tilde{\boldsymbol{\epsilon}}, \tilde{\boldsymbol{\epsilon}}>0$ for some small $\tilde{\boldsymbol{\epsilon}}$, a condition that also possesses a convex solution set. We observe the following lemma.

Contour Construction Lemma: Let $E^{\mathrm{R}+\mathrm{S}}$ be Euclidean $(R+S)$-dimensional space considered as a product of $E^{\mathrm{R}}$ and $E^{\mathrm{S}}$, with $\pi$ the projection onto $E^{\mathrm{R}}$. Let $U$ be an open set in $E^{\mathrm{R}+\mathrm{S}}$ with the following two properties:

(a) $U$ projects, under $\pi$, onto all of $E^{\mathrm{R}}$;

(b) The inverse image of each point under the map $\pi: u \rightarrow E^{\mathrm{R}}$ is a convex subset of $E^{\mathrm{s}}$.

Then there exists a differentiable (even $C^{\infty}$ ) cross section, i.e., there is a differentiable map $F: E^{\mathfrak{R}} \rightarrow U$ such that $\pi F: E^{\mathrm{R}} \rightarrow E^{\mathrm{R}}$ is the identity map.

Proof: Pick a point $[x, t(x)]$ in $U$ for each point $x$ in $E^{\mathrm{R}}$. Each such $[x, t(x)]$ is contained in a product neighborhood $u_{1}(x) \times u_{2}(x) \subset u$. In the neighborhood $u_{1}(x)$ of $x$ in $E^{\mathbf{R}}$, the map $f_{x}: y \rightarrow[y, t(x)]$ is a $C^{\infty}$ cross section. There is a locally finite refinement $V_{\alpha}$ of the $u_{1}(x)$ and a subordinate $C^{\infty}$ partition of unity $\phi_{\alpha}$. The convexity of the fibers allows this $C^{\infty}$ partition of unity to provide a global cross section:

where $V_{\alpha} \subset u_{1}[x(\alpha)]$.

$$
f=\sum_{\alpha} \phi_{\alpha} f_{x(\alpha)}
$$

This lemma enables us to construct a global contour of integration. $\left(1 / \lambda_{0}\right)$ is the $\eta$ of the theorem. For $0 \leq \theta \leq \eta$, the contour of integration may be chosen to be the momentum surface associated with $\lambda=1 / \theta$. (The lemma is applied with $E^{\mathbf{R}}$ the space of $\mathbf{x}$ sequences and $E^{\mathrm{S}}$ the space of $\mathrm{y}$ sequences.)

If the potentials have analyticity in a strip about real momentum values, and not in the full Yukawa region, the above construction must be modified to bound $\left|\mathbf{y}_{i}\right|$. We restrict ourselves to the following result.
Theorem 2: Let $\mathbf{k}_{\mathrm{in}}, \mathbf{R}_{\mathrm{fin}}$, and $\mathbf{I}_{\mathrm{fin}}$ be real vectors, with $\mathbf{R}_{\text {fin }}$ and $\mathbf{I}_{\text {fin }}$ satisfying the following conditions:

(a) $\left|\mathbf{I}_{\text {fin }}\right|<1$;

(b) If $\mathbf{k}_{\mathbf{0}}$ is any vector in the shell $(a-\delta \leq$ $\left|\mathbf{k}_{0}\right| \leq a+\delta$ ) and $\mathbf{R}_{\mathrm{fin}}-\mathbf{k}_{0}=\sum_{i \in J} \alpha_{i} \mathbf{a}_{i}$ for some subset indexed by $J$ of vectors from $P$, then $\mathbf{I}_{\mathrm{fin}}-\mathbf{k}_{0}=\sum_{i \in J} \beta_{i} \mathbf{a}_{i}$ for some $\beta_{i}$.

Then, given any $B>0$, there is a $\lambda_{0}$ such that the amplitude can be continued in the final momenta written as $\mathbf{k}_{\mathrm{fln}}=\mathbf{R}_{\mathrm{fin}}+i \lambda \mathbf{I}_{\mathrm{fin}}$ from $\lambda=0$ to $\lambda=\lambda_{\mathbf{0}}$, and $\left|\operatorname{Im} \mathbf{k}_{i}\right|<B$ for all intermediate stresses. $\lambda_{0}$ will depend on: (a) $M_{1}, M_{2}, \cdots, M_{N}$, (b) $N$, (c) $B$, (d) $\operatorname{Re} E$.

Proof: Let $Q$ be a sphere of radius greater than the maximum of $a+\delta$ and 1 , in the setup of the geometric theorem before. As before, solve the geometric problem for $\mathbf{x}_{1}=\mathbf{k}_{\mathrm{in}}$ and $\mathbf{x}_{\mathrm{fin}}=\mathbf{R}_{\mathrm{fin}}$. By Lemma 2 there is a sphere $Q^{\prime}$ greater than $Q$ such that if any subsequence of vectors in the solution set begin with a vector in $Q$ and end with a vector in $Q$, the solution set can be modified to keep all vectors inside $Q^{\prime}$. With such modifications this theorem follows from Theorem 1.

\section{CONCLUSION}

We indicate problems remaining to be studied. First, the behavior at infinity must be studied sufficiently to justify the contour distortion at infinity. Second, the equations for many-body scattering amplitudes should be continued similarly to the perturbation-theory case. The geometry of this problem seems treatable with only a slight generalization of the theorems included here. If the analytic questions relating to threshhold behavior and behavior at infinity for the integral equations can be treated systematically, a rigorous treatment of the problem of asymptotic completeness may be achieved. 